\title{
RADIAL VELOCITIES OF CLUSTERS BETWEEN THE MAGELLANIC CLOUDS
}

\author{
S. DEMERS AND L. GRONDIN $\dagger$ \\ Department of Physics \\ Université de Montréal, Montreal \\ Canada \\ M.J. IRWIN \\ Institute of Astronomy \\ Cambridge University, Cambridge \\ England \\ W.E. KUNKEL $\dagger$ \\ The Observatories of the Carnegie Institution of Washington \\ La Serena \\ Chile
}

ABSTRACT. Radial velocities of 20 stars members of young clusters and associations located in the inter-Cloud region within $2^{h}<R . A .<5^{h}$ tend to be lower than the HI velocities in the same direction. The stellar velocities do not show a pronounced velocity gradiant as observed for the HI from the SMC to the LMC. This suggests that the stellar components belong to the SMC wing. Our photometry and spectroscopy support the view that one of the stars at $4^{h} 20^{m}$ is a foreground HB star.

The young stellar aggregates identified between the Magellanic Clouds by Irwin, Demers \& Kunkel (1990) are believed to be debris resulting from the close encounter of the SMC with the LMC. They are relatively well aligned, stretching along the HI bridge mapped by McGee $\&$ Newton (1986). To better establish their origin, it would be of interest to determine if they share or not the motion of the HI across the bridge. McGee \& Newton (1986) have shown that clouds of well defined velocities occupy the bridge region. The velocity pattern is fairly obvious. Several groups of low radial velocity, $v \sim 200 \mathrm{~km} / \mathrm{sec}$ extend from the SMC wing and more tenuous high-velocity components, $v \sim 250 \mathrm{~km} / \mathrm{sec}$ are observed on the west side of the LMC. Because of their young age, it is more appropriate to compare the kinematic properties of the stellar groups with the HI rather than with intermediate-age objects, such as planetary nebulae or older systems like globular clusters. A close inspection of the isodensity contours of the HI reveals that the young clusters and associations do not necessary coincide with the peaks of HI density.

Several surveys of stellar radial velocities have been done in the past in the SMC wing and also in the inter-Cloud region. Those surveys have been impaired by the lack of information on the nature of the stars observed. A high-velocity star can be a LMC/SMC member or can also be a halo star. Without the knowledge of their absolute magnitude it may be nearly impossible to establish the true radial velocity pattern of the stars in this region.

In an attempt to answer these questions, we have, in the past few years, collected spectra and radial velocity of a number of bright early-type stars in and around the stellar

$\dagger$ Visiting astronomers, Cerro Tololo Inter-American Observatory, National Optical Astronomy Observatory, which is operated by the Association of Universities for Research in Astronomy Inc. under contract with the National Science Foundation. 
aggregates. A first series of velocities was published by Irwin, Demers \& Kunkel (1990). A second series was recently obtained at CTIO using the 2D-frutti attached to the Yale $1 \mathrm{~m}$ reflector. Even though the radial velocities of the candidates could be established with confidence using a cross correlation technique, the spectra were too noisy to be accurately classified. For all these stars, we have $\mathrm{V}$ magnitude and $\mathrm{B}-\mathrm{V}$ color, nearly evenly divided between CCD and photographic magnitudes.

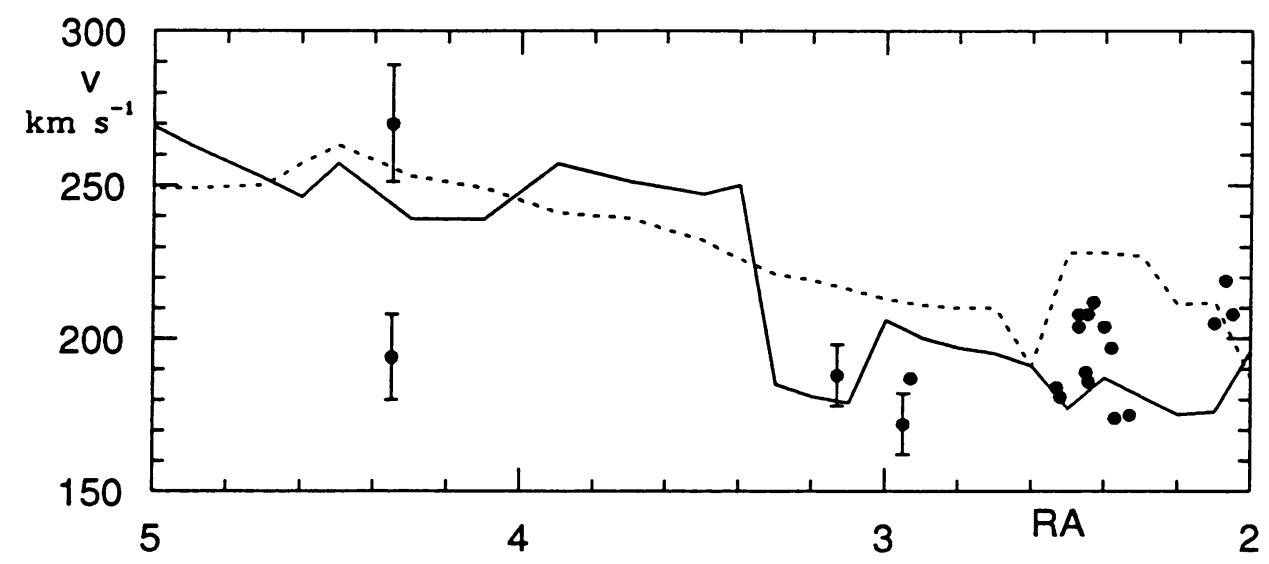

Figure 1. Average heliocentric HI velocities.

In order to compare the HI and stellar velocities, we display, in Figure 1, the average heliocentric HI velocities, (McGee \& Newton (1986) at $-73^{\circ}$ (dashed line) and at $-74^{\circ}$ (solid line). The dots represent the main sequence stars in young stellar aggregates. A few error bars are also shown. For most stars the error on the velocity is $\pm 10-20 \mathrm{~km} \mathrm{~s}^{-1}$.

A HI velocity gradiant is obvious; the velocities range from $\sim 190 \mathrm{~km} \mathrm{~s}^{-1}$ in the west to $\sim 260 \mathrm{~km} \mathrm{~s}^{-1}$ in the east. There are some low-velocity clouds east of $4^{h}$ but they count for only a few percents of the total HI density this can easily be seen on the $N_{H}$ maps of McGee \& Newton (1986). The velocities of the early-type stars match the low-velocity HI clouds, suggesting that the stars are kinematically associated with the SMC wing. The results for the two brightest stars in the association at $4^{h} 20^{m}$ require more comments.

For both stars we have $\mathrm{U}, \mathrm{B}, \mathrm{V}$ photometry. We can then estimate their temperature from the $\mathrm{Q}$ method. From the width of the Balmer lines and following Greenstein \& Sargent (1974), we are able to determine their $\log g$. The low-velocity star is indeed a main-sequence star. The $\log g$ of the high-velocity star, when matched to the data of Greenstein \& Sargent, is not incompatible with the gravity of a horizontal-branch star. One such foreground halo star, with similar radial velocity, was also found in the sample of Irwin, Demers \& Kunkel (1990). Several stars brighter than $\mathrm{V}=17$ in this association need to be observed in order to confirm our internretation.

\section{References}

Greenstein, J.L. and Sargent, A.I. (1974), Astrophys. J. Suppl. 28, 157.

Irwin, M.J., Demers, S. and Kunkel, W.E. (1990), Astron. J. 99, 191.

McGee, R.X. and Newton, L.M. (1986), Proc. Astron. Soc. Australia 6, 471. 